PROCEEDINGS OF THE

AMERICAN MATHEMATICAL SOCIETY

Volume 128, Number 10, Pages 2947-2956

S 0002-9939(00)05325-9

Article electronically published on April 7, 2000

\title{
UNIFORMLY BOUNDED MAXIMAL $\varphi$-DISKS, BERS SPACE AND HARMONIC MAPS
}

\author{
I. ANIĆ, V. MARKOVIĆ, AND M. MATELJEVIĆ \\ (Communicated by Albert Baernstein II)
}

\begin{abstract}
We characterize Bers space by means of maximal $\varphi$-disks. As an application we show that the Hopf differential of a quasiregular harmonic map with respect to strongly negatively curved metric belongs to Bers space. Also we give further sufficient or necessary conditions for a holomorphic function to belong to Bers space.
\end{abstract}

\section{INTRODUCTION}

Holomorphic quadratic differentials on a Riemann surface arise in several distinct areas of geometry, for instance in Teichmüller theory and in the theory of harmonic maps (see, for example, Ahlfors [2, Earle and Eells [3], Wolf [21], Jost [7]).

In the introduction we give a short review of our results as well as some related ones.

In $\S 1$ we use a special parameter (natural parameter) in terms of which the differential has a particularly simple representation, along with the theorems of Bloch and Koebe, to prove the following result:

Theorem 1. A holomorphic quadratic differential $\varphi d z^{2}$ on the unit disc is bounded with respect to the Poincaré metric (i.e. it belongs to Bers space) if and only if the radii of its maximal $\varphi$-discs are uniformly bounded.

After writing the previous version we realized that this result has its roots in known characterizations of Bloch functions in terms of their image Riemann surface. Pommerenke [11] proved that a function $f$ belongs to Bloch space if and only if its image surface $W_{f}$ contains no large schlicht discs. For relevant definitions related to this result and some generalizations we refer the interested reader to Stegenga and Stephenson [15].

Note that when we work with a natural parameter, we have some additional difficulties caused by possible zeroes of the corresponding quadratic differential. Lemma 1.1 (see below) enables us to overcome those difficulties.

We will mention some recent results, which motivated us.

Wan [20] proved that a harmonic diffeomorphism of the hyperbolic plane $\mathbb{H}^{2}$ is quasiconformal if and only if its Hopf differential is uniformly bounded with

Received by the editors April 20, 1998 and, in revised form, August 27, 1998 and November 18, 1998.

1991 Mathematics Subject Classification. Primary 30F30; Secondary 32G15, 58E20.

Key words and phrases. Quadratic differentials, Bers space, quasiregular harmonic maps, negatively curved metrics. 
respect to the Poincaré metric. This has also been generalized to hyperbolic CartanHadamard surfaces by Li, Tam and Wang [19]. See Tam and Wan [17], [18] and Han [5] for a general discussion of this area, where this and other questions were discussed.

As an application of Theorem 1 we show that the Hopf differential of a quasiregular harmonic map with respect to a strongly negatively curved metric belongs to Bers space (see below, Theorems 2 and 3 \$2).

Thus, roughly speaking, we can extend one direction of the above-mentioned characterizations ([20], 19]) of harmonic quasiconformal mappings to harmonic quasiregular mappings.

For a precise definition of quasiregular mapping see \$2, A4. Here we note only that the notion of quasiregular mapping is a natural generalization of the notion of a quasiconformal mapping since one does not require that quasiregular mappings be homeomorphisms.

Our proofs of Theorems 2 and 3 are based on the fact that the Bochner formula (see [13, [14, 17) has a simple form with respect to the natural parameter. This allows us to define a metric by means of the dilatation of the mapping, whose Gaussian curvature is bounded from above by -1 , and we use the classical AhlforsSchwarz lemma.

In $\$ 3$ we give further sufficient and necessary conditions for a holomorphic function to belong to Bers space, and show that every quasiregular harmonic mapping is decomposable as a quasiconformal harmonic mapping followed by an analytic function.

For further results and the literature in this growing area we refer the interested reader to [5, 6, 9, 10, 13, 17, 18, 19, 20, 21, 22.

We close our paper with a short discussion concerning some further results and open problems.

\section{Maximal $\varphi$-Disks And Bers SpaCe $Q$}

Let $\varphi$ be an analytic function on the unit disk $\Delta$. Then $\varphi$ belongs to Bers space $Q=Q(\Delta)$ if

$$
\operatorname{ess} \sup \omega(z)^{2}|\varphi(z)|<+\infty
$$

where $\omega(z)=1-|z|^{2}$.

In this section we will give a characterization of Bers space by means of maximal $\varphi$-disks (see Theorem 1 below). First we define maximal $\varphi$-disks.

Maximal $\varphi$-disk. Let $\varphi$ be an analytic function on the unit disk $\Delta$ and let $z_{0}$ be a regular point of $\varphi$, i.e. $\varphi\left(z_{0}\right) \neq 0$. Let $\Phi_{0}$ be a single valued branch of

$$
w=\Phi(z)=\int \sqrt{\varphi(z)} d z
$$

near $z_{0}, \Phi\left(z_{0}\right)=0$. There is a neighborhood $U$ of $z_{0}$ which is mapped one-to-one conformally onto an open set $V$ in the $w$-plane. We can assume, by restriction, that $V$ is a disk around $w=0$. The inverse $\Phi_{0}^{-1}$ is a conformal homeomorphism of $V$ into $\Delta$ and evidently there is a largest open disk $V_{0}$ around $w=0$, such that the analytic continuation of $\Phi_{0}^{-1}$ (which is still denoted by $\Phi_{0}^{-1}$ ) is homeomorphic and $\Phi_{0}^{-1}\left(V_{0}\right) \subset \Delta$. The image $U_{0}=\Phi_{0}^{-1}\left(V_{0}\right)$ is called the maximal $\varphi$-disk around $z_{0}$; its $\varphi$-radius (injectivity radius) $r_{0}$ is the Euclidean radius of $V_{0}$. 
For the definition of $\varphi$-disks and a discussion of their important role in the theory of holomorphic quadratic differentials we refer the interested reader to Strebel's book [16].

Bloch's and Koebe's theorems. The two following theorems play an important role in the proof of Theorem 1 . We need the following versions of Bloch's and Koebe's theorems.

Theorem A (Bloch). Let $w=f(z)$ be an analytic function on the disk $B=$ $B\left(z_{0}, r\right)=\left\{z:\left|z-z_{0}\right|<r\right\}, r>0$, and let $f^{\prime}\left(z_{0}\right) \neq 0$. Then there is an open disk $U$ together with an open set $V \subset B$ such that $f$ restricted to $V$ defines a one-to-one mapping of $V$ onto $U$ and the radius $R$ of $U$ satisfies

$$
R \geq C\left|f^{\prime}\left(z_{0}\right)\right| r
$$

where $C$ is an absolute constant.

Theorem $\mathbf{B}$ (Koebe). Let $V$ be a domain in $\mathbb{C}$, let $f$ be an analytic and univalent mapping which maps $V$ onto the disk $U=\left\{w:\left|w-w_{0}\right|<R\right\}$ and let $z_{0}=f^{-1}\left(w_{0}\right)$. Then

$$
\operatorname{dist}\left(z_{0}, \partial V\right)\left|f^{\prime}\left(z_{0}\right)\right| \geq \frac{R}{4}
$$

The following lemma enables us to use Bloch's theorem. In the proof of this lemma we will use the hyperbolic metric on a disk.

Hyperbolic distance. Let $B$ be the disk with center at $z_{0}$ and radius $r$. Using the conformal automorphisms $\phi_{a}(z)=\frac{z-a}{1-\bar{a} z}, a \in \Delta$, of $\Delta$, one can define pseudohyperbolic distance on $\Delta$ by

$$
\delta(a, b)=\left|\phi_{a}(b)\right|, a, b \in \Delta .
$$

Next, using the conformal map $A(\zeta)=\frac{\zeta-z_{0}}{r}$ from $B$ onto $\Delta$, one can define pseudo-hyperbolic distance on $B$ by

$$
\delta_{B}(z, w)=\delta(A(z), A(w))
$$

and the hyperbolic metric on $B$ by

$$
\rho(z, w)=\log \frac{1+\delta_{B}(z, w)}{1-\delta_{B}(z, w)}
$$

for $z, w \in B$.

The following result is well known.

Theorem C. Let $F$ be an analytic function from a disk $B$ to another disk $U$. Then $F$ does not increase the corresponding hyperbolic (pseudo-hyperbolic) distances.

Lemma 1.1. Let $\varphi$ be a bounded analytic function on the disk $B=B\left(z_{0}, r_{0}\right)$ and let $M_{0}=\sup \{|\varphi(z)|: z \in B\}$. Suppose that $\varphi\left(z_{0}\right) \neq 0$ and let $r_{1}=\frac{r_{0}}{M_{0}}\left|\varphi\left(z_{0}\right)\right|$. Then $\varphi$ has no zeroes in the disk $B\left(z_{0}, r_{1}\right)$.

Proof. Let $\varphi(z)=0$ for some $z \in B$. Using the hyperbolic (or pseudo-hyperbolic) distances on $B$ and $B\left(0, M_{0}\right)$, an application of Theorem $[$ to the analytic function $\varphi$ and the points $z$ and $z_{0}$ yields

$$
\frac{\left|z-z_{0}\right|}{r_{0}} \geq \frac{\left|\varphi\left(z_{0}\right)\right|}{M_{0}}
$$


Let $\varphi$ be an analytic function on the unit disk $\Delta$. Let $0<r<1$ and $\varphi_{r}(z)=$ $\varphi(r z) r^{2}, \psi(z)=\left|\varphi_{r}(z)\right|^{\frac{1}{2}}$ and $\omega(z)=1-|z|^{2}$. Assume that the function $h(z)=$ $\omega(z) \psi(z)$ has the maximum on $\Delta$ at the point $z_{0} \in \Delta$. Next let $r_{0}=\frac{1-\left|z_{0}\right|}{2}$ and let $M_{0}=\max \left\{\left|\varphi_{r}(z)\right|: z \in B\left(z_{0}, r_{0}\right)\right\}$. Since

$$
h\left(z_{0}\right) \geq h(z)
$$

and

$$
2 \omega(z) \geq \omega\left(z_{0}\right) \text { for } z \in B\left(z_{0}, r_{0}\right),
$$

then

$$
M_{0} \leq 4\left|\varphi_{r}\left(z_{0}\right)\right|
$$

An application of Lemma 1.1 to the disk $B\left(z_{0}, r_{0}\right)$ shows that $\varphi_{r}$ does not have zeroes in the disk $B\left(z_{0}, r_{1}\right)$, where $r_{1}=\frac{r_{0}}{M_{0}}\left|\varphi_{r}\left(z_{0}\right)\right|$. Next, by (1)

$$
r_{1} \geq \frac{r_{0}}{4}=r_{2} .
$$

Since $\varphi_{r}$ does not have zeroes in $B\left(z_{0}, r_{2}\right)=B$, there is a regular branch of the function $\sqrt{\varphi_{r}}$ in $B$, and therefore a regular branch $\Phi$ of $\int \sqrt{\varphi_{r}}$ in $B$. Since $\left|\Phi^{\prime}\left(z_{0}\right)\right|=$ $\sqrt{\left|\varphi_{r}\left(z_{0}\right)\right|}$, then, by Bloch's theorem, there is a disk $V$ of radius

$$
R=R\left(z_{0}\right) \geq C \psi\left(z_{0}\right) \cdot r_{2}
$$

where $C$ is an absolute constant, such that $\Phi^{-1}$ is univalent on $V$. Let $R_{\infty}=$ $\sup \left\{R_{z}: z \in \Delta\right\}$, where $R_{z}$ is the radius of the maximal $\varphi$-disk around $z$. Suppose that $R_{\infty}$ is finite. Then

$$
R_{\infty} \geq \frac{C}{8} \psi\left(z_{0}\right)\left(1-\left|z_{0}\right|\right) \geq \frac{C}{16} \psi\left(z_{0}\right) \omega\left(z_{0}\right) .
$$

When $r \rightarrow 1_{-}$, one can obtain that

$$
R_{\infty}^{2} \geq\left(\frac{C}{16}\right)^{2}\|\varphi\|,\|\varphi\|=\sup _{z \in \Delta} \omega^{2}(z)|\varphi(z)| .
$$

Lemma 1.2. Suppose that $\varphi \in Q$. Then $R_{\infty}$ is finite.

Proof. Let $\Delta_{z}$ be the maximal $\varphi$-disk around $z \in \Delta$ and let $R_{z}$ be the euclidean radius of the disk $\Phi\left(\Delta_{z}\right)$, where $\Phi$ is the natural parameter. By Koebe's Theorem

$$
\operatorname{dist}\left(z, \partial \Delta_{z}\right)\left|\Phi^{\prime}(z)\right| \geq \frac{R_{z}}{4} .
$$

Since $1-|z| \geq \operatorname{dist}\left(z, \partial \Delta_{z}\right)$ and $\left|\Phi^{\prime}(z)\right|=\sqrt{|\varphi(z)|}$ then

$$
\|\varphi\| \geq \frac{R_{\infty}^{2}}{16} .
$$

The following result is an immediate corollary of (2) and Lemma 1.2

Theorem 1. Let $\varphi$ be an analytic function on $\Delta$. Then $\varphi \in Q$ iff $R_{\infty}$ is finite. 


\section{Harmonic maps and Bers space $Q$}

Harmonic maps play an important role in the parametrization of Teichmüller spaces (see Earle and Eells [3] and Wolf [21]), so it is interesting to understand the relation between universal Teichmüller space and quasiconformal harmonic diffeomorphisms. For further results see Wan [20, Tam and Wan [17], Reich and Strebel [12. In this direction we have the following result (the terminology will be explained, and the proof given, later in this section).

Theorem 2. Let $\rho$ be the metric with Gaussian curvature $K \leq-a$ for some constant $a>0$, and let $f$ be a harmonic quasiregular map from $\Delta$ into itself with respect to $\rho$. Then the Hopf differential $\varphi$ of $f$ belongs to $Q$.

Theorem 2 is an immediate corollary of Theorem 1 and Lemma 2.1 See below for the proof of this lemma and for the definition of a quasiregular function.

Let $R$ and $S$ be two surfaces. Let $\sigma(z)|d z|^{2}$ and $\rho(w)|d w|^{2}$ be the metrics with respect to the isothermal coordinate charts on $R$ and $S$ respectively, and let $f$ be a $C^{2}$-map from $R$ to $S$.

It is convenient to use notation in local coordinates $d f=p d z+q d \bar{z}$, where $p=f_{z}$ and $q=f_{\bar{z}}$. Also we introduce the complex (Beltrami) dilatation

$$
\mu_{f}=\operatorname{Belt}[f]=\frac{q}{p}
$$

where it is defined.

The energy integral of $f$ is

$$
E(f, \rho)=\int_{R} \rho \circ f\left(|p|^{2}+|q|^{2}\right) d x d y .
$$

A critical point of the energy functional is called a harmonic mapping. The Euler-Lagrange equation for the energy functional is

$$
\tau(f)=f_{z \bar{z}}+(\log \rho)_{w} \circ f p q=0 .
$$

Thus, we say that a $C^{2}$-map $f$ from $R$ to $S$ is harmonic if $f$ satisfies the above equation. For basic properties of harmonic maps and for further information on the literature we refer to Jost [7] and Schoen-Yau [13].

The following facts and notation are important in our approach:

(A1). If $f$ is a harmonic mapping, then

$$
\varphi d z^{2}=\rho \circ f p \bar{q} d z^{2}
$$

is a quadratic differential on $R$, and we say that $\varphi$ is the Hopf differential of $f$ and we write $\varphi=\operatorname{Hopf}(f)$.

(A2). The Gaussian curvature on $S$ is given by

$$
K_{S}=-\frac{1}{2} \frac{\Delta \log \rho}{\rho} .
$$

(A3). We will use the following notation: $\mu=\operatorname{Belt}[f]=\frac{q}{p}$ and $\tau=\log \frac{1}{|\mu|}$ and the Bochner formula (see [13])

$$
\Delta \tau=-K_{S}|\varphi| \sinh \tau
$$


(A4) Definition of quasiregular function. Let $R$ and $S$ be two Riemann surfaces and let $f: R \rightarrow S$ be a $C^{2}$-mapping. If $P$ is a point on $R, \tilde{P}=f(P) \in S, \phi$ a local parameter on $R$ defined near $P$ and $\psi$ a local parameter on $S$ defined near $\tilde{P}$, then the map $w=h(z)$ defined by $h=\left.\psi \circ f \circ \phi^{-1}\right|_{V}(V$ is a sufficiently small neighborhood of $P$ ) is called a local representer of $f$ at $P$. The map $f$ is called $k$-quasiregular if there is a constant $k \in(0,1)$ such that for every representer $h$, at every point of $R,\left|h_{\bar{z}}\right| \leq k\left|h_{z}\right|$.

Lemma 2.1. Let $\rho$ be the metric on $\Delta$ with Gaussian curvature $K$ uniformly bounded from above on $\Delta$ by the negative constant $-a$, and let $f$ be a harmonic $k$-quasiregular map from $\Delta$ into itself with respect to the metric $\rho$. If $R=R_{z}$ is the radius of the maximal $\varphi$-disk around $z$, where $\varphi=\operatorname{Hop} f(f)$, then $R$ is bounded from above by the constant $C$ which depends only on $k$ and $a$.

Proof. Let $R=R_{z}$ be the radius of the maximal $\varphi$-disk $U=U_{z}$ around $z \in \Delta$. Since $f$ is $k$-quasiregular then $\tau \geq m$, where $m=\log \frac{1}{k} . m>0$. Let $\zeta=\Phi(z)$ be the natural parameter in $U$ and $\Phi(U)=V=B(0, R)$. With respect to the parameter $\zeta$ the Bochner formula takes the simple form

$$
\Delta \tau=-K \sinh \tau .
$$

Since $K \leq-a$ and $\tau \geq m$, we conclude that

$$
\Delta \tau \geq \delta e^{\tau} \text { on } V
$$

where $\delta=\frac{a \sinh m}{e^{m}}$. Let $d s=\lambda(\zeta)|d \zeta|$, where $\lambda(\zeta)=\frac{2 R}{R^{2}-|\zeta|^{2}}$ is the hyperbolic metric on $V$ and let $\tilde{\lambda}(\zeta)=\left(\frac{\delta}{2} e^{\tau(\zeta)}\right)^{\frac{1}{2}}$. From (4) we have for the Gaussian curvature of the metric $d \tilde{s}=\tilde{\lambda}(\zeta)|d \zeta|$ on $V$ that $\tilde{K} \leq-1$, and then we can use the Ahlfors-Schwarz Lemma (see [1]) to obtain

$$
\frac{\delta}{2 k} \leq \tilde{\lambda}^{2}(\zeta) \leq \lambda^{2}(\zeta)
$$

Setting $\zeta=0$ in (5) one obtains

$$
R^{2} \leq \frac{8 k}{\delta}
$$

Let $\varphi$ be a quadratic differential on a hyperbolic Riemann surface $R$ with Poincaré metric $d s^{2}=\rho(z)|d z|^{2}$. Let $p \in R$ and let $z$ be a local parameter near $p$. We will define

$$
\|\varphi\|(p)=\rho^{-1}(z(p))|\varphi(z(p))| .
$$

We say that $\varphi$ belongs to the Bers space of $R$ (notation $Q(R)$ ) if $\|\varphi\|$ is a uniformly bounded function on $R$.

Theorem 3. Let $R$ and $S$ be hyperbolic surfaces with metric densities $\sigma$ and $\rho$ respectively and let the Gaussian curvature of the metric $d s^{2}=\rho(w)|d w|^{2}$ be uniformly bounded from above on $S$ by the negative constant $-a$. If $f$ is a harmonic $k$-quasiregular map from $R$ into $S$ with Hopf differential $\varphi$, then $\varphi \in Q(R)$.

Proof. Let $\tilde{f}$ be the lifting of $f$ which maps $\Delta$ into itself and let $\tilde{\varphi}$ be the lifting of the quadratic differential $\varphi$. Let $\tilde{\rho}$ be the lifting of the density $\rho$. Since $\tilde{f}$ is harmonic with respect to the metric $\tilde{\rho}(\tilde{w})|d \tilde{w}|^{2}$ on $\Delta$ and $k$-quasiregular then, by Theorem 2, $\tilde{\varphi} \in Q(\Delta)$. Hence $\varphi \in Q(R)$. 


\section{Further RESUlts}

In Theorem 4 we will give a characterization of a quasiregular harmonic map.

Theorem 4. Let $f$ be a k-quasiregular harmonic map from $\Delta$ into itself with respect to some metric $d s^{2}=\rho(w)|d w|^{2}$. Then $f=F \circ g$, where $F$ is an analytic function from $\Delta$ into itself and $g$ is a $k$-quasiconformal mapping from $\Delta$ onto itself, which is harmonic with respect to the metric $d \tilde{s}^{2}=\tilde{\rho}(\zeta)|d \zeta|^{2}$, where $\tilde{\rho}=\rho \circ F\left|F^{\prime}\right|^{2}$.

Proof. Since $f$ is harmonic on $\Delta$ then $\varphi=\rho \circ f p \bar{q}$ is an analytic function on $\Delta$. Therefore $p$ has isolated zeroes or $p$ is identically 0 on $\Delta$. If $p \equiv 0$ on $\Delta$, then $q \equiv 0$ and $f \equiv$ const on $\Delta$ and our theorem is trivial. If $p$ has isolated zeroes on $\Delta$, then we can define $\mu=\frac{q}{p}$ a.e. on $\Delta$.

It is known that there is a quasiconformal mapping $g$ from $\Delta$ onto itself such that $g$ is a solution of the Beltrami equation

$$
g_{\bar{z}}=\mu g_{z}
$$

(see [2], 8]).

Let $F=f \circ g^{-1}$. Then we have for $\operatorname{Belt}[F]$ (see [2], [8]) that

$$
\mu_{F} \circ g=\frac{g_{z}}{\bar{g}_{\bar{z}}} \cdot \frac{\mu_{f}-\mu_{g}}{1-\mu_{f} \bar{\mu}_{g}}=0,
$$

and we conclude that $F$ is an analytic function.

Since $f$ is harmonic with respect to $\rho$ then

$$
\varphi(z)=\rho(f(z)) p \bar{q}
$$

is an analytic function in $\Delta$, where $p=f_{z}$ and $q=f_{\bar{z}}$. Since $p(z)=F^{\prime}(\zeta) A(z)$ and $q(z)=F^{\prime}(\zeta) B(z)$, where $A=g_{z}, B=g_{\bar{z}}$ and $\zeta=g(z)$, one can obtain that

$$
\varphi(z)=\tilde{\rho}(\zeta) A \bar{B}
$$

Since $g$ is quasiconformal $|A| \neq|B|$ a.e. and $\varphi_{\bar{z}} \equiv 0$ on $\Delta$, one can show that $\tau(g)=0$ (for computation of $\varphi_{\bar{z}}$ see, for example, Jost [7] and Tam-Wan [18]).

Let $D$ be a hyperbolic domain in $\mathbb{C}, z \in D$, and let $d s=\rho(z)|d z|$ be the corresponding hyperbolic metric on $D$. Then it is known that (Theorem 1.11, [1])

$$
\rho(z) \leq \frac{2}{r(z)}, z \in D,
$$

where $r(z)=\operatorname{dist}(z, \partial D)$, and we call $r(z)$ the distance function (see [1]).

For an analytic function $\varphi$ on a domain $D \subset \mathbb{C}$ we say that $\varphi \in \tilde{Q}$ if

$$
\operatorname{ess} \sup \operatorname{dist}^{2}(z, \partial D)|\varphi(z)|<\infty \text {. }
$$

Since the distance function is geometrically simpler than the hyperbolic density, it is reasonable to study the space $\tilde{Q}$.

We say that a domain $D \subset \mathbb{C}$ is strongly hyperbolic if it is hyperbolic and diameters of boundary components are uniformly bounded from below by a positive constant.

Theorem 5. Let $D$ be a strongly hyperbolic and bounded domain in $\mathbb{C}$. Then $\varphi \in \tilde{Q}$ iff $\varphi \in Q$. 
Proof. Because of (7) we have that $Q \subset \tilde{Q}$. Let the diameters of boundary components of $D$ be bounded from below by $d>0$, and let the diameter of $D$ be equal to $M$.

Now let $z \in D$. We can find a component $l$ of $\partial D$ for which $r(z)=\left|z-z_{0}\right|$, where $z_{0} \in l$. Let $\tilde{D}=\overline{\mathbb{C}} \backslash l$ and let $\rho$ and $\tilde{\rho}$ be the corresponding hyperbolic linear densities of $D$ and $\tilde{D}$ respectively. Since $D \subset \tilde{D}$ then $\tilde{\rho}(z) \leq \rho(z)$ for $z \in D$ (see [1]).

Let $\tilde{r}(z)=\operatorname{dist}(z, \partial \tilde{D})$, and let $c \in l$ such that $\left|z_{0}-c\right|=\frac{d}{2}$. The function $\psi(\zeta)=\frac{1}{\zeta-c}$ maps $\tilde{D}$ conformally onto the domain $G \subset \mathbb{C}$. Since $G$ is conformally equivalent to the unit disk, by the Koebe Theorem

$$
\sigma(w) \geq \frac{1}{4\left|w-w_{0}\right|}
$$

where $w=\psi(z), w_{0}=\psi\left(z_{0}\right)$ and $\sigma$ is the linear density of hyperbolic metric on $G$.

From (8) we can conclude that

$$
\tilde{\rho}(z) \geq \frac{\left|z_{0}-c\right|}{|z-c|} \cdot \frac{1}{\left|z-z_{0}\right|}
$$

and hence

$$
\tilde{\rho}(z) \geq \frac{C}{\tilde{r}(z)}
$$

where $C=\frac{d}{d+2 M}$.

Since $\tilde{r}(z)=r(z)$ we finally obtain that

$$
\rho(z) \geq \frac{C}{r(z)} .
$$

Hence $\tilde{Q} \subset Q$.

The next example shows that if the boundary of a domain $D$ has a point as a component, then the spaces $Q$ and $\tilde{Q}$ are different.

Example 1. Let $D$ be $\Delta \backslash\{0\}$, and $\varphi(z)=\frac{1}{z^{2}}$. It is obvious that $\varphi \in \tilde{Q}$. The linear density of the hyperbolic metric on $D$ is

$$
\rho(z)=\frac{1}{|z| \log \frac{1}{|z|}} .
$$

Then

$$
\rho^{-2}(z)|\varphi(z)|=\log \frac{1}{|z|}
$$

which is not bounded in $D$; hence $\varphi \notin Q$.

In fact, any function

$$
\varphi(z)=\frac{1}{z^{2}} \psi(z)
$$

where $\psi$ is an analytic function in $\Delta$ with $\psi(0) \neq 0$, is not in $Q$.

Example 2. Let $D$ be $\mathbb{C} \backslash[-1,1]$. Then $\psi(w)=\frac{1}{2}\left(w+\frac{1}{w}\right)$ is a conformal mapping from $\Delta \backslash\{0\}$ onto $D$. Let $\varphi$ be an analytic function on $D$. It is clear that $\varphi \in Q(D)$ iff $\varphi_{1} \in Q(\Delta \backslash\{0\})$, where

$$
\varphi_{1}(w)=\varphi(z)\left(\psi^{\prime}(w)\right)^{2}
$$


Let $\varphi(z)=\frac{1}{z^{2}}$. It is obvious that $\varphi \in \tilde{Q}(D)$. Since

$$
\varphi_{1}(w)=\frac{1}{w^{2}} \frac{1}{2}\left(\frac{w^{2}-1}{w^{2}+1}\right)^{2}
$$

then, by Example[1, we conclude that $\varphi_{1} \notin Q(\Delta \backslash\{0\})$; hence $\varphi \notin Q(D)$.

Using Koebe's Theorem as in Lemma 1.2 one can prove the following result:

Proposition 3.1. Let $D$ be a hyperbolic domain in the complex plane $\mathbb{C}$. If $\varphi \in$ $\tilde{Q}(D)$, then the radii of the maximal $\varphi$-disks are uniformly bounded on $D$.

As we mentioned in the Introduction we close with a short discussion of some further results in this area.

Wolf [22] and Minsky [10] have shown that estimates on the dilatation of a harmonic map depend to a great extent on the geometry of the Hopf differential $\varphi$ (in particular, on the placement of the zeroes of $\varphi$ and the injectivity radius in the $\varphi$-metric).

Han [5] and Han, Tam, Treibergs and Wan 6] have used the Wolf-Minsky type estimates mentioned above to study among other things the images of harmonic diffeomorphisms of $\mathbb{C}$ into the hyperbolic plane $\mathbb{H}$.

We believe that our results can be of use in understanding some parts of this interesting area, as well as being of interest in their own right.

\section{ACKNOWLEDGEMENT}

We wish to thank the referee for numerous useful comments and suggestions which greatly improved the exposition of our paper and for pointing out that our results can possibly be of use in a number of popular open problems. We also would like to thank M. Wolf for useful conversations and his interest in this work, A. Baernstein for pointing out to us the Stegenga-Stephenson paper 15 and for helping us to prepare this paper, C.V. Stanojević for his continous encouragement and support during the last few years and B. Hanson for his interest in this work and suggestions concerning the language.

\section{REFERENCES}

[1] Ahlfors, L., Conformal invariants, McGraw-Hill Book Company, 1973. MR 50:10211

[2] Ahlfors, L., Lectures on Quasiconformal Mappings, Van Nostrand, 1966. MR 34:336

[3] Earle, C.J. and Eells, J., A Fibre bundle description of Teichmüler theory, J. Diff.Geom. 3 (1969) 19-43. MR 43:2737a

[4] Gardiner, F.,P., Teichmüller Theory and Quadratic Differentials, New York: WileyInterscience Publication, 1987. MR 88m:32044

[5] Han, Z-C., Remarks on the geometric behavior of harmonic maps between surfaces, Elliptic and parabolic methods in geometry. Proceedings of a workshop, Minneapolis, May 23-27, 1994, Wellesley. MR 98a:58048

[6] Han, Z-C., Tam, L-F., Treibergs, A. and Wan, T., Harmonic maps from the complex plane into surfaces with nonpositive curvature, Commun.Anal.Geom. 3 (1995) 85-114. MR 96k:58057

[7] Jost, J., Two-dimensional Geometric Variational Problems, John Wiley \& Sons, 1991. MR 92h:58045

[8] Lehto, O. and Virtanen, K.I., Quasiconformal Mappings in the Plane, Springer-Verlag, 1973. MR 49:9202

[9] Marković, M. and Mateljević, M., New versions of Reich-Strebel inequality and uniqueness of harmonic mappings, to appear.

[10] Minsky, Y., Harmonic maps, length and energy in Teichmüller space, J. Diff. Geom. 35 (1992), 151-217. MR 93e:58041 
[11] Pommerenke, Ch., On Bloch functions, J. London Math. Soc. (2), 2 (1970), 689-695. MR 44:1799

[12] Reich, E. and Strebel, K., On the Gerstenhaber-Rauch principle, Israel J.Math. 57 (1987) 89-100. MR 88g:30028

[13] Schoen, R. and Yau, S.,T., Lectures on Harmonic Maps, Conf. Proc. and Lect. Not. in Geometry and Topology, Vol.II, Inter. Press, 1997. MR 98i:58072

[14] Schoen, R. and Yau, S.,T., On univalent harmonic maps between surfaces, Invent.Math. 44 (1978), 265-278. MR 57:17706

[15] Stegenga, D. and Stephenson, K., A geometric characterization of analytic functions with bounded mean oscillation, J. London Math. Soc. (2), 24 (1981), 243-254. MR 82m:30036

[16] Strebel, K., Quadratic Differentials, Springer-Verlag, 1984. MR 86a:30072

[17] Tam, L. and Wan, T., Quasiconformal harmonic diffeomorphism and universal Teichmüler space, J.Diff.Geom. 42 (1995) 368-410. MR 96j:32024

[18] Tam, L. and Wan, T., Harmonic diffeomorphisms into Cartan-Hadamard surfaces with prescribed Hopf differentials, Comm. Anal. Geom. 4 (1994) 593-625. MR 96m:58057

[19] Li, P., Tam, L. and Wang, J., Harmonic diffeomorphisms between hyperbolic Hadamard manifolds, Jour. Geom. Anal., to appear.

[20] Wan, T., Constant mean curvature surface, harmonic maps, and universal Teichmüller space, J. Diff. Geom. 35 (1992) 643-657. MR 94a:58053

[21] Wolf, M., The Teichmüller theory of harmonic maps, J.Diff.Geom. 29 (1989) 449-479. MR 90h:58023

[22] Wolf, M., High-energy degeneration of harmonic maps between surfaces and rays in Teichmüler space, Topology 30 (1991), 517-540. MR 92j:32075

Faculty of Mathematics, University of Belgrade, Studentski Trg 16, Belgrade, YuGOSLAVIA

E-mail address: ianic@matf.bg.ac.yu

School of Mathematics, University of Minnesota, Minneapolis, Minnesota 55455

E-mail address: markovic@math.umn.edu

Faculty of Mathematics, University of Belgrade, Studentski Trg 16, Belgrade, YuGOSLAVIA

E-mail address: miodrag@matf.bg.ac.yu 